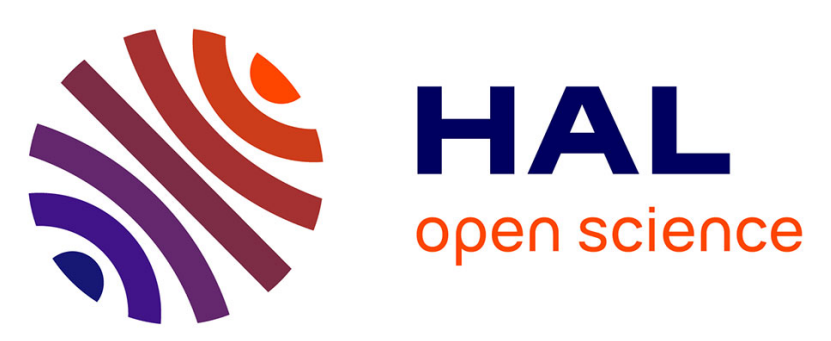

\title{
Impact of MAC Scheduling on Positioning Accuracy for Motion Capture with Ultra Wideband Body Area Networks
}

Arturo Guizar, Anis Ouni, Claire Goursaud, Nicolas Amiot, Jean-Marie Gorce

\section{- To cite this version:}

Arturo Guizar, Anis Ouni, Claire Goursaud, Nicolas Amiot, Jean-Marie Gorce. Impact of MAC Scheduling on Positioning Accuracy for Motion Capture with Ultra Wideband Body Area Networks. BodyNet 2014, Sep 2014, London, United Kingdom. 10.4108/icst.bodynets.2014.257113 . hal-01096503

\section{HAL Id: hal-01096503 https://hal.science/hal-01096503}

Submitted on 17 Dec 2014

HAL is a multi-disciplinary open access archive for the deposit and dissemination of scientific research documents, whether they are published or not. The documents may come from teaching and research institutions in France or abroad, or from public or private research centers.
L'archive ouverte pluridisciplinaire HAL, est destinée au dépôt et à la diffusion de documents scientifiques de niveau recherche, publiés ou non, émanant des établissements d'enseignement et de recherche français ou étrangers, des laboratoires publics ou privés. 


\section{Impact of MAC Scheduling on Positioning Accuracy for Motion Capture with Ultra Wideband Body Area Networks}

\author{
Arturo Guizar \\ University of Lyon \\ INSA-Lyon, CITI-INRIA \\ 69100, Villeurbanne, France \\ arturo.guizar@insa- \\ lyon.fr
}

\author{
Anis Ouni \\ Telecom ParisTech \\ 23 avenue d'Italie \\ 75014, Paris, France \\ anis.ouni@telecom- \\ paristech.fr
}

\author{
Claire Goursaud \\ University of Lyon \\ INSA-Lyon, CITI-INRIA \\ 69100, Villeurbanne, France \\ claire.goursaud@insa- \\ lyon.fr
}

\author{
Nicolas Amiot \\ University of Rennes 1, IETR \\ 263 Avenue Général Leclerc \\ 35042 Rennes, France \\ nicolas.amiot@univ- \\ rennes1.fr
}

Jean-Marie Gorce

University of Lyon

INSA-Lyon, CITI-INRIA

69100, Villeurbanne, France

jean-marie.gorce@insa-

lyon.fr

\begin{abstract}
Wireless Body Area Networks (WBANs) have recently emerged as a solution to enable effective location-aware applications. The rapid mobility of nodes is the most specific problem of this kind of networks and it is not well considered when designing MAC protocols. In this paper, we consider a WBAN using a typical TDMA-based Medium Access Control (MAC) protocol and an Impulse Radio Ultra Wideband (IR-UWB) physical layer defined by the standard IEEE802.15.6. We investigate the impact of mobility on the Motion Capture applications. The Root Mean Square Error (RMSE) of the estimated positions is analyzed according to different scheduling strategies at MAC layer under a real human mobility model. Our results show that an effective scheduling scheme leads to prioritize the own node position estimation. Finally, we propose to extend the study with a realistic human body channel.
\end{abstract}

\section{Categories and Subject Descriptors}

C.2.1 [Network Architecture and Design]: Wireless communication; D.3.3 [Network Architecture and Design]: System de-sign issues, and performance modelling; H.1.2 [Models and Principles]: Miscellaneous

\section{General Terms}

Experimentation, Performance, Measurement

\section{Keywords}

Wireless Body Area Networks, Ultra WideBand, Localisation, Motion Capture, IEEE 802.15.6, Scheduling

\section{INTRODUCTION}

Wireless Body Area Networks (WBANs) are a core enabling technology that define "the Internet of you" which is the new brand of the Internet of things with the potential to change the way people go about their daily lives, specially in health care system. In this context, WBANs have raised the interest of researchers to find innovative solutions for e-Health applications $[1,2]$ such as emergency and rescue, disease detection or prevention, sport personal coaching, or personal multimedia. The latter can be achieved by estimating accurately the individual positions/postures with Motion Capture.

Individual Motion Capture systems can be classified in vision and non-vision techniques. The vision approach consist in a group of cameras capable to collect 3D location data of human motion i.e. Kinect, Vicon [3]. However, vision systems can not be used in all kinds of environments, as they do need lighting and line of sight. On the contrary, WBANs can be used to monitor the human body movement, either with sensors (i.e. accelerometers, gyroscopes, ...) or distance estimation based algorithms. In this case, WBANs need to address several challenges such as energy efficiency, accurate precision, low complexity and reliable communication. For this purpose, Impulse Radio Ultra Wideband (IR-UWB) systems [4] are a good solution to estimate precise range measurements using Time of Arrival (TOA) algorithm which is based on the transmission of packets : a request, and 1 or 2 responses. When considering a mobile target, the actual positions may be different for each packet. This may increase the error distance estimation, in particular, when the time between packets transmission increases. Thus, the scheduling is of primary importance. In order to give an effective location, the MAC layer must take into account the rapid mobility of WBAN nodes. In this paper, we tackle the impact of mobility on the position estimation accuracy and motion capture application. Different scheduling strategies are proposed and compared in order to give some engineering useful insights to design effective MAC protocols for location-aware applications. 
The rest of the paper is organized as follows. Section 2 reviews the related work that focus on localization problems for motion capture. Then, in Section 3, we give the problem statement and the system model, and we explain our real mobility model for individual motion capture application. In Section 4, we present the proposed scheduling strategies to reduce the positioning error. Section 6 study the impact of mobility on location-aware applications. Finally, we conclude the paper in Section 7.

\section{RELATED WORK}

Extensive works of research on Individual Motion Capture have present the challenges to consider for localization IRUWB systems, such as clock synchronization, NLOS environments (Non Line of Sight), interference mitigation and multipath [5]. Nevertheless, they do not consider the MAC scheduling impact on ranging estimation to reduce the positioning error under mobility for WBAN scenarios. In [6], they present the issues of ranging error, position update latency and calculation algorithms under mobility. They show the impact of MAC allocation resources on the capacity of the tracking system for Wireless Sensor Networks (WSN) scenarios.

Moreover, previous works focusing on MAC design for localization with UWB systems proposed protocol strategies based on beacon-enabled Time Division Multiple Access and evaluated the performance in terms of accuracy and latency. In [7], they proposed cooperative ranging with Aggregated and Broadcast schemes to reduce the delay of $3 \mathrm{WR}$ transactions. In [8], they focus on better resource management with priority levels for communication. In [9], they focus on the relation between MAC delay and UWB accuracy related to the number of anchors and the communication range of nodes under mobility. However, all these works focuses on localization applications for WSN which do not present the same problems of WBAN and they do not consider the impact of the scheduling of localization packets on the positioning estimation. In [10], they proposed scheduling schemes for cooperative distributed localization with two different policies (node neighbourhood and links quality) to reduce positioning convergence, latency and overhead. However, they consider a 2D Positioning for WSN which again is not realistic for WBAN location-aware applications.

Furthermore, the recent works in localization with WBAN have been focusing on the radio issues and localization algorithms performances without a rigorous scope on MAC strategies. In [11], the authors modelled the ranging error in terms of TOA estimation with real IR-UWB channel measurements in order to perform better localization algorithms. In [12], they realize a realistic measurement setup to achieve accurate positioning of WBAN nodes and comparing the results with a vicon system [3].

\section{ASSUMPTIONS AND SYSTEM MODEL}

\subsection{Network Topology}

We consider a mesh IR-UWB WBAN under full connectivity containing $N_{T}$ nodes of two kinds as in [11], on-body mobile nodes that do not know their own position $(i=1 \ldots N)$ and on-body anchor nodes that know their own position $(j=1 \ldots M), N_{T}=N+M$. A set of anchor nodes de- fine a Local Coordinate System (LCS) to localize nodes under mobility. We define the instantaneous distance of the node $\mathrm{i}$ with the anchor $\mathrm{j}$ as $d_{i j}(t)$ and the estimated distance as $\hat{d}_{i j}(t)$ which is calculated through TOA estimation. Moreover, the instantaneous position for a node is defined as $P_{i}(t)$ and the estimated position is defined as function of estimated distances $\hat{P}_{i}(t)=f\left(\hat{d}_{i 1}(t), \hat{d}_{i 2}(t), \ldots, \hat{d}_{i N}(t)\right)$.

Finally, a set of $N_{T}$ positions $P(t)=\left\{P_{1}(t), \ldots, P_{N_{T}}(t)\right\}$ at time $t$ define a human body posture at the LCS and the estimated posture is defined as the set of estimated positions $\hat{P}(t)=\left\{\hat{P}_{1}(t), \ldots, \hat{P}_{N_{T}}(t)\right\}$.

\subsection{Localisation System Model}

As presented in Section 1, we use the IR-UWB systems for the motion capture to estimate the positions and distances between the nodes in real time. The distance $\hat{d}_{i j}(t)$ between two nodes is deduced with the Three-Way Ranging (3WR) protocol by combining the typical timers obtained from 3 transmissions [6], corresponding to one Request $\left(Q_{i j}\right)$ send by a node $\mathrm{i}$ to an anchor $\mathrm{j}$ and two Response packets $\left(R_{i j}\right.$ and $S_{i j}$ ) from anchor $\mathrm{j}$ to node i, as shown in Figure 1. We define $\Delta t 1$ (resp. $\Delta t 2$ ) as the time difference between the reception of a request packet and the transmission of a response 1 packet (resp. the time difference between the transmission of the responses packets).

$$
\hat{d}_{i j}(t)=\frac{1}{2} c\left[\left(\left(T_{4}-T_{1}\right)-\Delta t 1\right)-\left(\left(T_{6}-T_{4}\right)-\Delta t 2\right)\right]
$$

where $c$ is the light speed.

The position $\hat{P}_{i}(t)$ of a node is estimated with the Time Difference of Arrival (TDOA) technique [4]. For this purpose, each node should communicate with at least four anchors, which is the minimum needed for a tridimensional positioning.

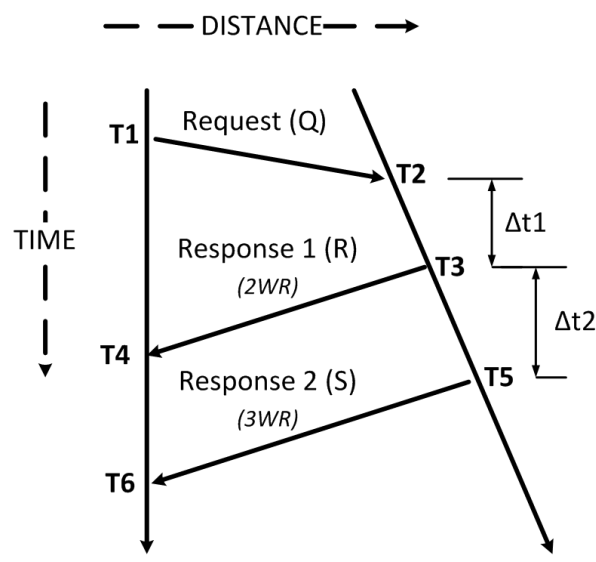

Figure 1: 2WR and 3WR protocols

\section{PROPOSED SCHEDULING STRATEGIES}

As explained in Section 3.1, it is necessary to estimate accurate distances for positioning the nodes of a WBAN in a LCS in order to enable the human motion capture. To this aim, we define a Medium Access Control (MAC) Layer based on the TDMA protocol and we assume that it is beacon enabled. Then, we reserve three transmission periods corresponding to the $3 \mathrm{WR}$ protocol. For the sake of our 
study, the scheduling of the $3 \mathrm{WR}$ periods is flexible to allow the comparison of different scheduling strategies (Section 4) to reduce the ranging error. In this study, we have to consider that the accuracy of the $3 \mathrm{WR}$ protocol depends on the delay taken to receive the response packets which varies when we have several nodes in the network, therefore, the ranging error estimated with the TOA may increase for some nodes.

To illustrate this assumption, we can take our previous scenario presented in [13] where one node is sending a request for ranging estimation with an anchor, as shown in Figure 1. As the node is always moving, it is evident that the distance between the node and the anchor at time $T 1$ may be different to the distance at $T 6$, thus, there is a ranging error related to the time taken to receive the last response, considering the distance at $T 1$ as the one we want to estimate. From this study, we find that the time to send the first response $\Delta t 1$ has more impact on ranging estimation than $\Delta t 2$. Moreover, we find the same problem for the positioning, if we consider the case of one node sending $3 \mathrm{WR}$ packets to four anchors in order to find its position as in Figure 2, it is straightforward that the position of the node may not be the same between the moment it send the first request packet and at the moment it receive the response packets of the last anchor. This positioning error can be reduced by proposing the most appropriate scheduling scheme at the MAC layer. In this section, we define different scheduling strategies to minimize the ranging and positioning error.

\subsection{Scheduling for accurate positioning}

First, we define different strategies in order to reduce the positioning error a node $i$ helped by $j$ anchors in a WBAN. We consider that a node calculate its estimated position $\hat{P}_{i}(t)$ when he has estimated the distances $\hat{d}_{i j}(t)$ of at least four anchors. These schemes are shown in Figure 2

All request first (S1): The request packets $Q_{i j}$ are sent in priority to all the anchors. Then each anchor answers at its turn with a Response $1(\mathrm{R})$ and a Response 2 (S) consecutively. Ordered transaction (S2): The node starts a 3WR transaction with each anchor in order. Three period order (S3): The MAC Frame is divided into three periods dedicated to send the different $3 \mathrm{WR}$ packets in order: the $Q_{i j}$, the $R_{i j}$ and the $S_{i j}$ at the end. Priority for Response 1 (S4): The node send the Request and the anchor send a Response 1 packet immediately. Then, the anchors send the $S_{i j}$ at the end of the frame.

These four schemes have been selected because they all have their own advantage that can lead to a better accuracy. Indeed, $\mathrm{S} 1$ increases $\Delta t 1$ and $\Delta t 2$, therefore, the ranging error on the estimated distances is also increased but these will be more closed to the estimated position. S2 reduces $\Delta t 1$ and $\Delta t 2$ to give a more accurate distances, however they will be far from the last position. S3 reduces $\Delta t 1$ which is the most important for ranging estimation while increases $\Delta t 2$. S4 reduces highly $\Delta t 1$ and increases $\Delta t 2$. Thus, the goal of this study is to identify the best strategy.

\subsection{Scheduling schemes for motion capture}

In this second study, we focus on the motion capture. The goal is to locate precisely and simultaneously several mobile

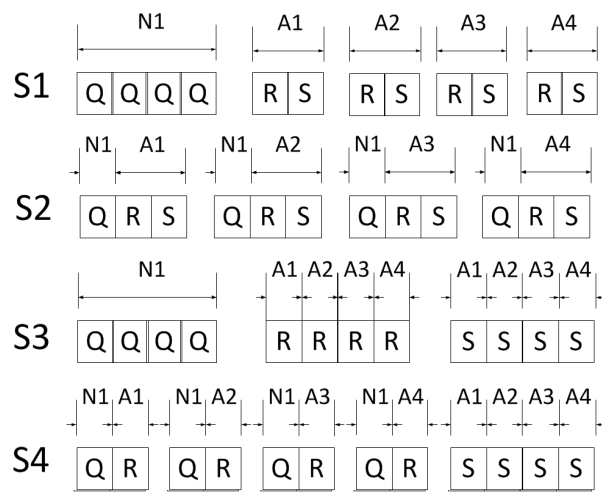

Figure 2: Scheduling strategies for 1 node and 4 anchors

nodes to identify the posture of the person of interest. In this case, there is a tradeoff between having all distances corresponding to the same posture, and having these distances with high accuracy. To this aim, we propose the following strategies:

All request first, anchor priority (S5): The nodes send the $Q_{i j}$ packets at the beginning, then each anchor answer in order to all nodes with $R_{i j}$ and $S_{i j}$. All request first, node priority (S6): As the previous, the nodes send $Q_{i j}$ first then all anchors answer node by node with $R_{i j}$ and $S_{i j}$. Ordered node positioning (S7): Each node repeat the scheme S1 one by one.

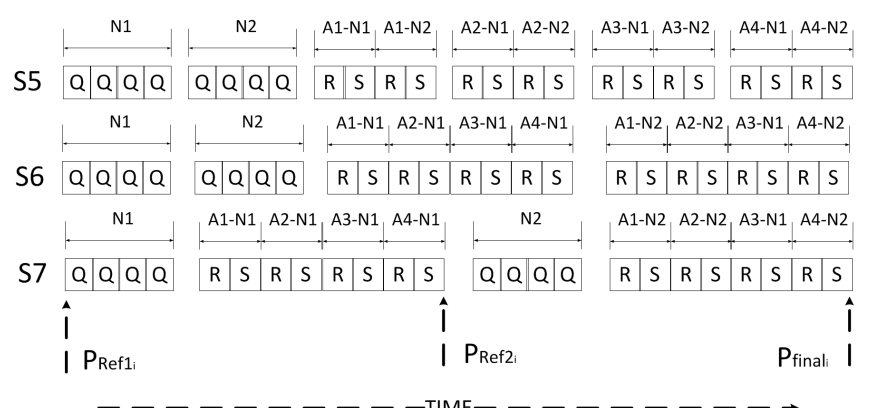

Figure 3: Scheduling strategies for 2 nodes and 4 anchors

\section{SIMULATION FRAMEWORK \\ 5.1 Simulation tools and parameters}

In our study, we adopt a discrete-event simulation approach using the WSNet simulator [14] which allows to test different communication protocols under realistic situations, specially for the upper layers. Thus, we can reuse the data collected from the Mobility Model (Section 5.2) to study the impact of mobility on ranging estimation as in our previous study [13]. This simulator is also suited for WBAN applications in terms of flexibility and simulation time, such as the motion capture. Moreover, it has a precision of time events in the nanoseconds order. Therefore, it is possible to study the performance of the $3 \mathrm{WR}$ protocol.

At the physical (PHY) Layer, we defined a protocol based on the IEEE802.15.6 PHY UWB [15] in default mode (OOK modulation, data rate $0.4875 \mathrm{Mbps}$ ). For the sake of simplicity, we assume a Line of Sight (LOS) channel without 
packet loss in order to focus on the ranging error related to the mobility and the MAC scheduling. Therefore, we assume that our radio is capable to detect the first path of IR-UWB to detect the precise TOA at the receiver.

\subsection{Mobility Model}

We have considered in this study 2 kinds of mobility model. The first and simplest one is a periodic linear movement (i.e. walking) at different speeds $(1-20 \mathrm{~m} / \mathrm{s})$. The anchors stay fixed during the simulation and the node moves in a linear space of $30 \mathrm{~cm}$.

Besides, we have also considered a more realistic one based on real measurements. The mobility traces used in this paper have been obtained from the CORMORAN measurement campaign which has been realized during the project June 2014 at ENS Cachan Bretagne, France. This campaign has consisted in a simultaneous capture of bodies movements, radio devices positions and radio traces along different scenarios compliant with the 2 main applications targeted into the CORMORAN ANR project: large scale individual motion capture (LSIMC) and coordinated group navigation (CGN).

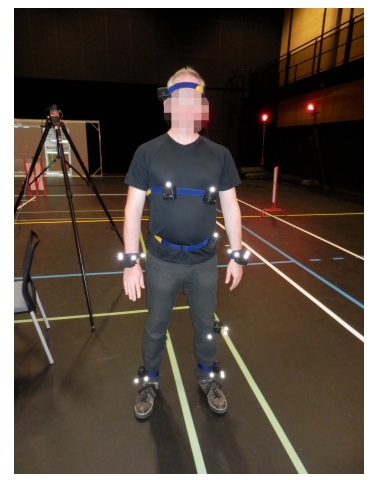

(a)

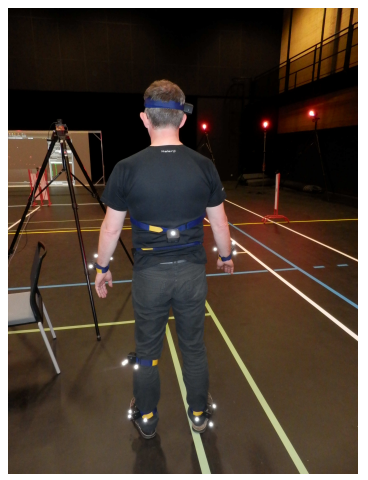

(b)
Figure 4: Pictures of the subject from (a) front and (b) back, equipped with the 10 TCR radio nodes. Shining dots on the TCR devices are the markers allowing to determine the position of radio nodes using the Vicon system.

The human body movements have been captured on an area of $10 \times 6$ meters using an ultra high precision system based on a Vicon Mx40 motion capture technology [3] at $100 \mathrm{~Hz}$. The system records the position of markers with the help of 16 cameras and is able to generate a C3D (Coordinates 3D) motion capture file containing the position of all the markers. Three different radio devices have been used along the scenarios of the measurement campaign: 1 IR-UWB "BeSpoon Phone" [16] and 2 tags providing distance observables, up to 10 IR-UWB "TCR" Devices from CEA LETI [17] providing Real time-Time Of Flight (RT-TOF) and up to 16 IEEE 802.15.4 "HikoB" Devices [18], providing power measurements. In this study, only the Vicon data have been used.

This paper focuses on a specific LSIMC scenario where a subject is performing static postures, and the solely radio device position information is used in order to feed the WSNet simulator [14]. In this scenario, the subject were equipped
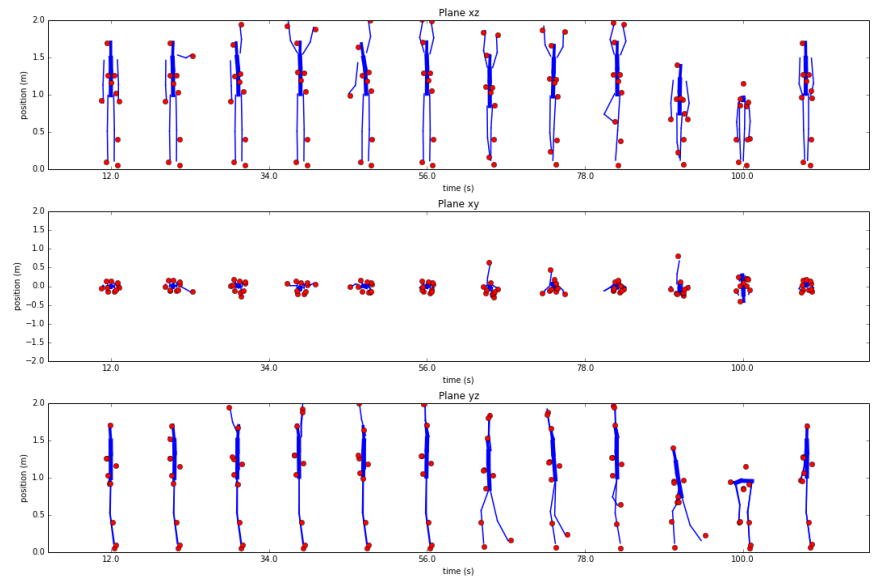

Figure 5: Decomposed body movement of the considered scenario along 3 different axes as a function of time. For this representation, the body position is sampled every 10 seconds along the complete 112 seconds scenario. Red dots correspond to the radio nodes positions.

with 66 markers: 41 markers was used for the motion capture and 25 markers were used for positioning radio devices. The position of the 41 markers dedicated to the motion capture have been chosen in order to disambiguate the upcoming body reconstruction. The radio devices located on globally static body parts (chest, back or hip ) was equipped with a single marker to determine their positions. The other devices located on moving body parts (arms or legs) was equipped with 4 markers in order to determine their orientation in addition of their positions. The positions of those marker on the body can be observed in Figure 4.

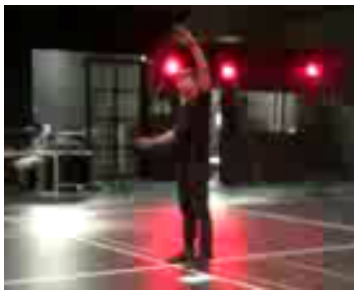

(a)

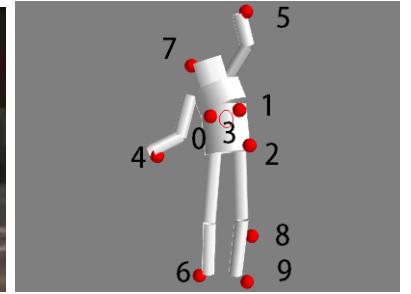

(b)
Figure 6: (a) Snapshot of the video camera record and (b) the associated multi-cylinders reconstructed body. The red dots represent the radio devices positions.

The first step of this work was to extract the needed data, and generate the mobility model used in WSNet. To this aim, with the help of the open source PyLayers platform [19] and especially its associated body module [20], it is possible to use the generated C3D file for visualizing the body movement, create an associated multi-cylinders body model and export the radio node positions. Hence, from the marker positions, a multi-cylinders body model is build to ensure the correct reconstruction of the body and of the radio nodes positions. Figure 6 compares a snapshot from a camera video shot and the associated multi-cylinders reconstruction, where the red dots represent the radio devices positions, and Figure 5 shows that feature by representing the different position of the body and of the radio nodes as a 
function of time. At this point, it is directly possible to export a WSNet compliant file containing the timestamped radio nodes positions. Then, with a dedicated mobility model implemented in WSNet, it is possible to simulate the mobility of nodes and evaluate the performance of the upper layer protocols.

\section{RESULTS}

\subsection{Impact on Positioning estimation}

In this section, we present the positioning error related to the scheduling schemes as proposed in Section 4.1. First, in order to test our system model, we consider a controlled scenario composed of a WBAN with five sensors: one node (right wrist) and four anchors (chest, back, hip right, hip left) under a periodic linear movement (i.e. walking) at different speeds $(1-20 \mathrm{~m} / \mathrm{s})$. We quantify the positioning error with the Root Mean Square Error (RMSE) - eq.2 by comparing the estimated position $\hat{P}_{i}(t)$ with the real position $P_{\text {final }_{i}}(t)$ at the end of the frame:

$$
R M S E=\sqrt{\frac{\sum^{N_{f}}\left|P_{\text {final }_{i}}(t)-\hat{P}_{i}(t)\right|^{2}}{N_{f}}}
$$

$N_{f}$ is the number of frames during the simulation.

Then, we extend the study with the mobility traces from Section 5.2 by using the node on the right wrist (4) and four anchors on Right Front Chest (0), Left Front Chest (1), Left hip (2) and Back Chest (3) as shown in Figure 6. We use a speed factor (1-10) in order to accelerate the movement traces of the node.

Figure 7 and 8 show that RMSE increases with the speed for all the strategies. This is due to the fact that, as the speed increases, the distance covered during $3 \mathrm{WR}$ transmissions is higher, inducing more errors in the distance estimation. Besides, for both Figures, strategies S1 and S2 give better positioning estimation than S3 and S4. In [13], we proved that increasing $\Delta t 1$ has more impact on the ranging error while increasing $\Delta t 2$ has an impact on the reference distance we want to estimate, in our scenario we compare the estimated position with the real position at the end of the frame $P_{\text {final }_{i}}(t)$. Thus, S1 and S2 are more efficient as they keep a reduced $\Delta t 2$ while in S3 and S4 it is increased. Moreover, we have completed this study by considering broadcast transmissions. In this case, the request is sent only once for all anchors, leading to a gain of Gain $_{\text {Brodcast }}=(M-1) * N$, i.e. 3 slots in our case. We can observe in Figures 7 and 8 that the broadcast on strategy S1 permits to reduce both error on positioning estimation and delay.

\subsection{Performance of scheduling strategies for motion capture}

In this second scenario, we evaluate the scheduling strategies proposed in Section 4.2 in terms of positioning error under the mobility model presented in Section 5.2. For this purpose, we keep the anchors at the same position, while the nodes to locate are on right wrist (4), left wrist (5), right ankle (6) and right head (7), as shown in Figure 6. As in the last study, we use a speed factor to accelerate the movement of nodes. We use the RMSE to compare the position estimated $\hat{P}_{i}(t)$ with a reference position, thus, we define three

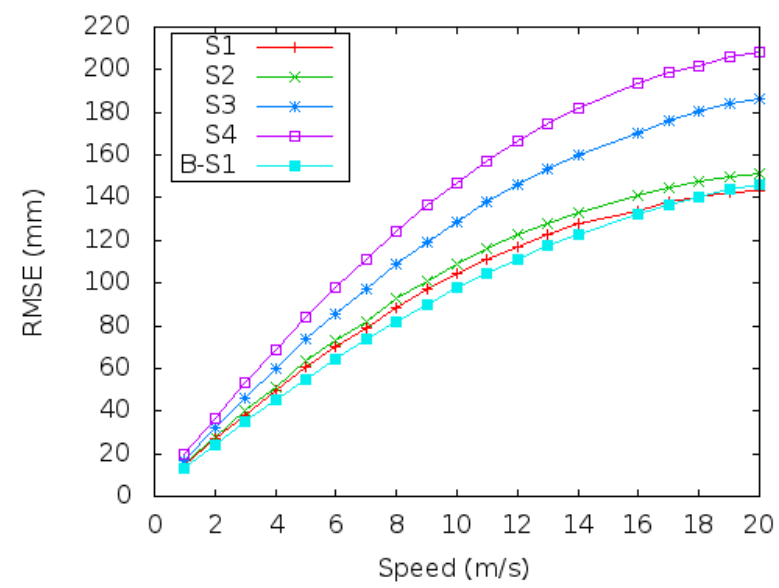

Figure 7: Impact of Scheduling under linear mobility

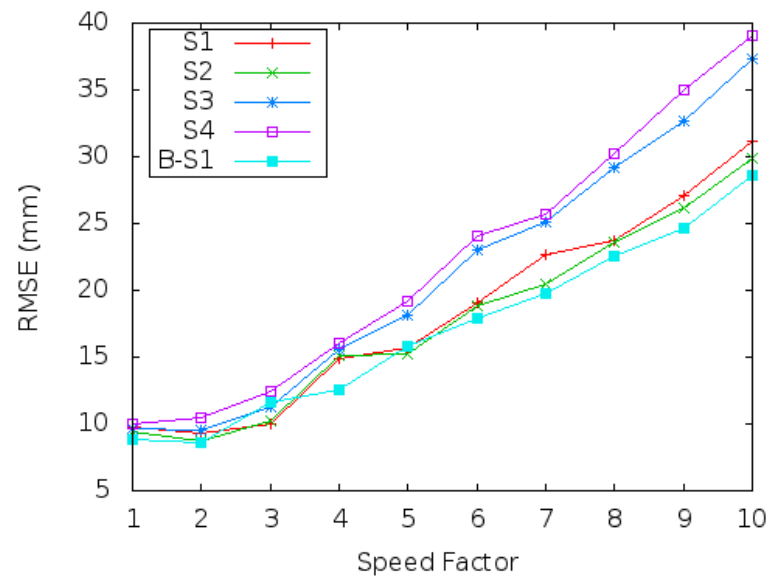

Figure 8: Impact of Scheduling under a realistic human mobility

reference positions for the node i : $P_{\operatorname{Ref1} 1_{i}}$ is the position of the node at beginning of a MAC frame, $P_{\operatorname{Ref} 2_{i}}$ is the instantaneous position at the end of the last $3 \mathrm{WR}$ transaction with all anchors and $P_{\text {final }}$ is the position of the node at the end of the frame, as shown in Figure 3.

Figure 9 shows that in the case of node 4 (right wrist), S7 is the best strategy to estimate $P_{R e f 1_{i}}$ and $P_{R e f 2_{i}}$ while S5 is better to estimate $P_{\text {final }_{i}}$. Figure 10 shows that in the case of node 5 (left wrist), S7 is a good choice to estimate $P_{R e f 2_{i}}$, but without remarkable difference $(1 \mathrm{~cm})$ for estimating $P_{R e f 1_{i}}$ and $P_{\text {final }_{i}}$ in respect to S5 and S6. Moreover, for nodes 4 and 5 , the estimation of $P_{\text {final }}$ has a more important error than the estimation of $P_{\operatorname{Ref} 1_{i}}$ and $P_{\operatorname{Ref2} 2_{i}}$. Figure 11 shows that in the case of node 6 (right ankle), S7 is the best strategy to estimate $P_{R e f 2_{i}}$ and $P_{f_{i n a l}}$. However in the case of the estimation of $P_{R e f 1_{i}}$ there is a small difference between S5, S6 and S7 (2mm). Figure 12 shows that in the case of node 7 (right head), S7 is the best strategy to estimate $P_{R e f 2_{i}}$ and $P_{\text {final }}$, while S5 is better to estimate $P_{R e f 1_{i}}$. Moreover, when applying a Broadcast transmission for the request packets for the best strategies of each scenario, Figures show an improvement (1-2cm) for all cases, but in this case we find a gain of 12 slots.

Note that when using S5, we have an averaged estimate of 
the positions between the start and the end of the frame. In the other side, S7 permits to estimate more accurate instantaneous positions. Therefore, nodes performing a position estimation at start of the frame (i.e. node 4) will have a better estimation of $P_{\text {final }}$ with S5, while the nodes at the end of the frame (i.e. 7) will find better estimation of $P_{\text {final }_{i}}$ with S7. We report the results on Table 1, the columns represent the position to estimate and the rows represent the best scheduling strategies to use depending on the scheduling of the node in the MAC frame.

Nodes scheduled at

\begin{tabular}{|c|c|c|c|}
\cline { 2 - 4 } \multicolumn{1}{c|}{ X of Frame } & $P_{\text {Ref } 1_{i}}$ & $P_{\text {Ref2 }_{i}}$ & $P_{\text {final }_{i}}$ \\
\hline Start & B-S7 & B-S7 & B-S5 \\
\hline Middle & B-S7 & B-S7 & B-S7 \\
\hline End & B-S5 & B-S7 & B-S7 \\
\hline
\end{tabular}

Table 1: Best Scheduling Strategy for Motion Capture

Based on these results, we find that in the case of an application looking for individual motion capture of the nodes (estimation of $P_{R e f 2_{i}}$ ) with high refreshment rate, the best scheduling is a Broadcast transmission with S7. If we enlarge the scope to applications looking for posture recognition (estimation of positions at the end (resp. start) of frame $P_{\text {final }}$ (resp. $\left.P_{R e f 1_{i}}\right)$, it is possible with a Broadcast transmission with S5.

\section{CONCLUSION AND DISCUSSION}

In this paper, we have addressed the problem of ranging error brought by the mobility of WBAN nodes to enable an Individual Motion Capture application with IR-UWB systems. First, we defined the architecture and workflow of the cross layer simulator, coupling "physical" blocks from PyLayers for the mobility model with the WSNet network simulation tool to create a dedicated simulator for Body Area Networks scenarios. Then we proposed different scheduling strategies in order to reduce the positioning estimation. We find that the best strategy in terms of positioning precision is the case B-S7 when each node realize a complete Broadcast $3 \mathrm{WR}$ transaction with all anchors to calculate their positions one by one. Next step is to enlarge the study with channel models under Realistic short-term and long-term pedestrian mobility models.

\section{ACKNOWLEDGEMENTS}

This work has been carried out in the frame of the CORMORAN project, which is funded in part by the French National Research Agency (ANR) under the contract number ANR-11-INFR-010.

\section{REFERENCES}

[1] S. Ullah, M. Mohaisen, and M. A. Alnuem, "A review of IEEE 802.15.6 MAC, PHY, and security specifications," International Journal of Distributed Sensor Networks, pp. 1-12, 2013.

[2] M. Hamalainen, A. Taparugssanagorn, and J. Iinatti, "On the WBAN radio channel modelling for medical applications," in Antennas and Propagation (EUCAP), Proceedings of the 5th European Conference on, pp. 2967-2971, 2011.

[3] "Vicon." http://www.vicon.com/.
[4] Z. Xiao, Y. Hei, Q. Yu, and K. Yi, "A survey on impulse-radio UWB localization," Sci. China Inf. Sci., vol. 53, pp. 1322-1335, Jul 2010.

[5] H. Soganci, S. Gezici, and H. Poor, "Accurate positioning in ultra-wideband systems," IEEE Wireless Communications, vol. 18, no. 2, pp. 19-27, April 2011.

[6] J. Choliz, A. Hernandez, and A. Valdovinos, "A framework for UWB-based communication and location tracking systems for wireless sensor networks," Sensors, vol. 11, pp. 9045-9068, Sep 2011.

[7] D. Macagnano, G. Destino, F. Esposito, and G. Abreu, "MAC performances for localization and tracking in wireless sensor networks," 2007 4th Workshop on Positioning, Navigation and Communication.

[8] M. Maman, B. Denis, and L. Ouvry, "An intuitive prioritised medium access scheme for tracking applications in UWB ldr-lt networks," 2008 IEEE 19th International Symposium on Personal, Indoor and Mobile Radio Communications.

[9] G. E. Garcia, L. S. Muppirisetty, and H. Wymeersch, "On the trade-off between accuracy and delay in UWB navigation," IEEE Commun. Lett., vol. 17, no. 1, pp. 39-42.

[10] B. Denis, M. Maman, and L. Ouvry, "On the scheduling of ranging and distributed positioning updates in cooperative IR-UWB networks," 2009 IEEE International Conference on Ultra-Wideband.

[11] J. Hamie, B. Denis, R. D'Errico, and C. Richard, "On-body toa-based ranging error model for motion capture applications within wearable UWB networks," Journal of Ambient Intelligence and Humanized Computing, Dec 2013.

[12] R. Bharadwaj, S. Swaisaenyakorn, C. G. Parini, J. Batchelor, and A. Alomainy, "Localization of wearable ultrawideband antennas for motion capture applications," Antennas Wirel. Propag. Lett., vol. 13, pp. 507-510, 2014.

[13] A. Guizar, A. Ouni, and C. Goursaud, "Impact of mobility on ranging estimation using ultrawideband," in Proceedings of the Fourth Networking Networking Women Workshop - N2Women ACM SIGCOMM, Chicago, USA, 2014.

[14] G. Chelius, A. Fraboulet, and E. Ben Hamida, "http://wsnet.gforge.inria.fr/."

[15] "IEEE standard for local and metropolitan area networks - part 15.6: Wireless body area networks," 2012. IEEE Std 802.15.6-2012.

[16] "Spoonphone." http://spoonphone.com/fr/.

[17] M. Pezzin and D. Lachartre, "A low power, low data rate impulse radio ultra wide band transceiver," in Future Network and Mobile Summit, 2010, pp. 1-10, June 2010.

[18] "Hikob." http://www.hikob.com/.

[19] N. Amiot, M. Laaraiedh, and B. Uguen, "Pylayers: An open source dynamic simulator for indoor propagation and localization," in Communications Workshops (ICC), 2013 IEEE International Conference on, pp. 84-88, June 2013.

[20] M. Mhedhbi, N. Amiot, S. Avrillon, and B. Uguen, "Human body perturbed antenna integration in indoor propagation simulator," 


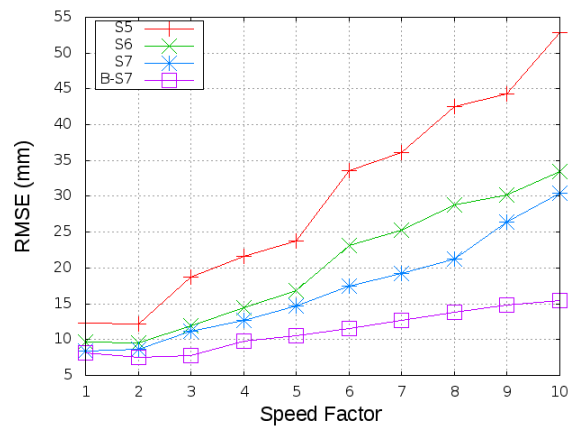

(a)

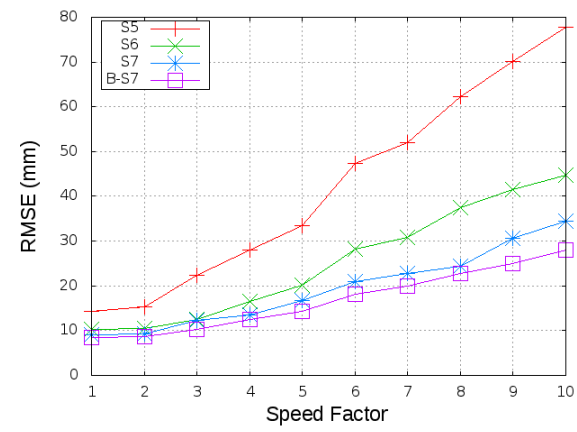

(b)

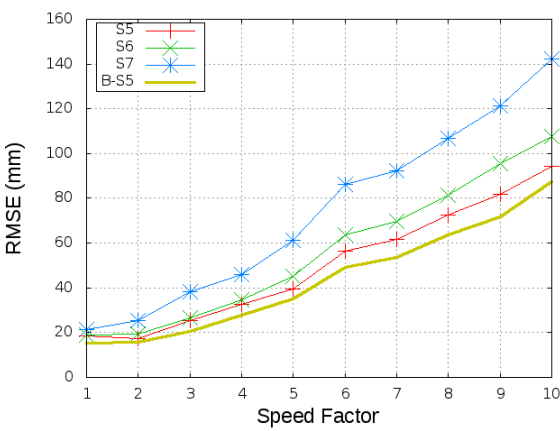

(c)

Figure 9: (a) Position error of node 4 respect to $P_{R e f 1_{i}}$. (b) Position error of node 4 respect to $P_{R e f 2_{i}}$. (c) Position error of node 4 respect to $P_{\text {final }_{i}}$.

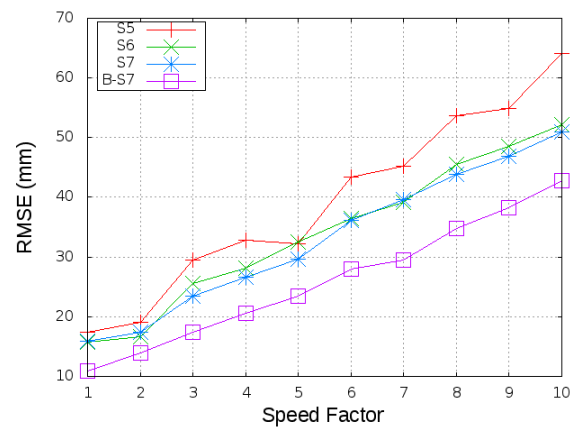

(a)

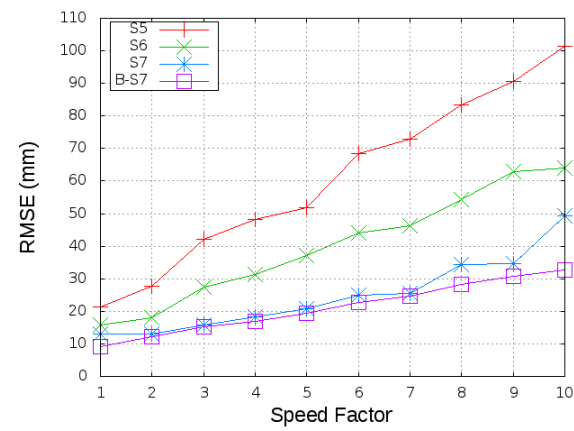

(b)

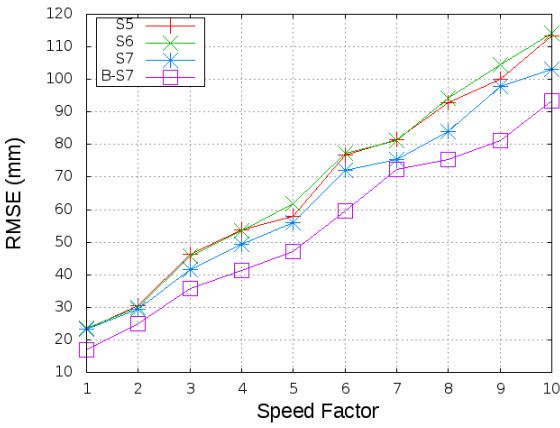

(c)

Figure 10: (a) Position error of node 5 respect to $P_{R e f 1_{i}}$. (b) Position error of node 5 respect to $P_{R e f 2_{i}}$. (c) Position error of node 5 respect to $P_{\text {final }_{i}}$.

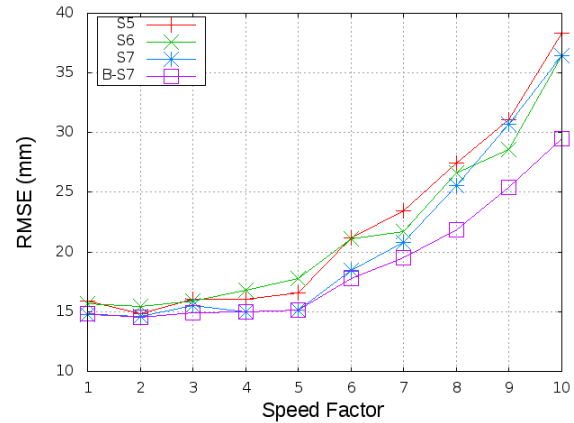

(a)

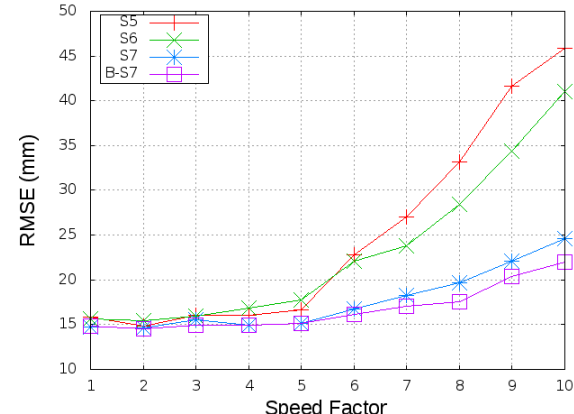

(b)

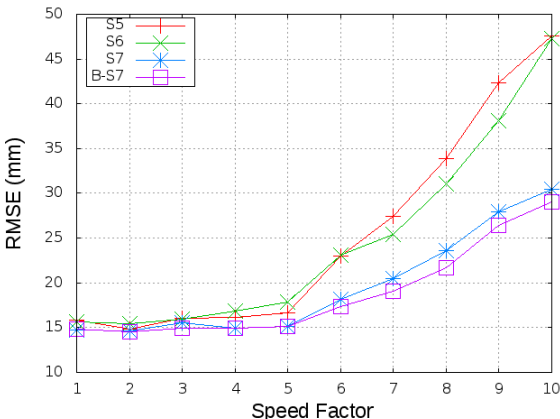

(c)

Figure 11: (a) Position error of node 6 respect to $P_{\operatorname{Ref} 1_{i}}$. (b) Position error of node 6 respect to $P_{\operatorname{Ref} 2_{i}}$. (c) Position error of node 6 respect to $P_{\text {final }_{i}}$.

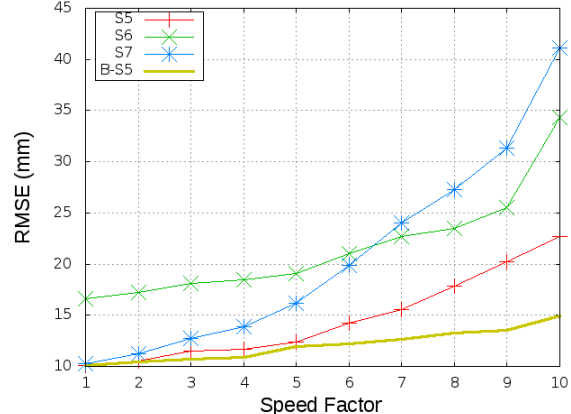

(a)

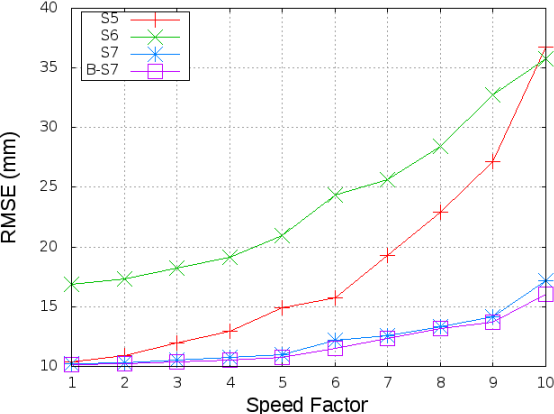

(b)

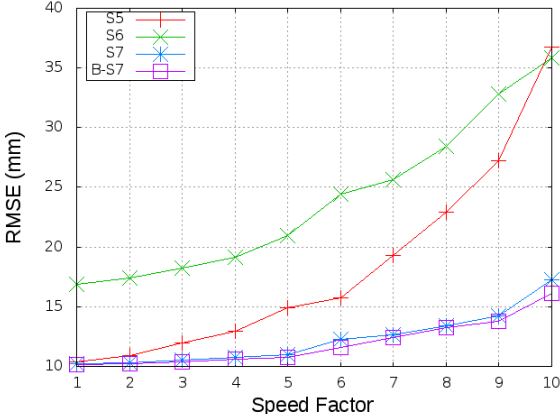

(c)

Figure 12: (a) Position error of node 7 respect to $P_{\operatorname{Ref1} 1_{i}}$. (b) Position error of node 7 respect to $P_{\operatorname{Ref2} 2_{i}}$. (c) Position error of node 7 respect to $P_{\text {final }_{i}}$. 